\title{
Single-Column Emulation of Reanalysis of the Northeast Pacific Marine Boundary Layer
}

${ }^{1}$ Department of Atmospheric Sciences, University of Washington, Seattle, Washington, USA.
J. McGibbon ${ }^{1}$ and C. S. Bretherton ${ }^{1}$

\section{Key Points:}

- A new machine learning boundary layer parameterization, MARBLE, is developed from ERA5 reanalysis data for the summertime Northeast Pacific.

- Used in a single-column model, MARBLE reproduces ERA5 thermodynamic structure and cloud properties over 7-day simulation periods.

- MARBLE reproduces the regional climatology of the stratocumulus to cumulus transition. 


\section{Abstract}

An artificial neural network is trained to reproduce thermodynamic tendencies and boundary layer properties from ERA5 HIRES reanalysis data over the summertime Northeast Pacific stratocumulus to trade cumulus transition region. The network is trained prognostically using 7-day forecasts rather than using diagnosed instantaneous tendencies alone. The resulting model, Machine Assisted Reanalysis Boundary Layer Emulation (MARBLE), skillfully reproduces the boundary layer structure and cloud properties of the reanalysis data in 7-day single-column prognostic simulations over withheld testing periods. Radiative heating profiles are well-simulated, and the mean climatology and variability of the stratocumulus to cumulus transition are accurately reproduced. MARBLE more closely tracks the reanalysis than does a comparable configuration of the underlying forecast model.

\section{Introduction}

Stratocumulus clouds cover over one-fifth of Earth's surface in the annual mean (Warren et al., 1986). These clouds strongly reflect incoming shortwave radiation and have little effect on outgoing longwave radiation, resulting in a strong cooling radiative effect on Earth's energy balance (Chen et al., 2000; Wood, 2012). Small changes in stratocumulus cover and thickness are sufficient to produce radiative effects comparable to the effects associated with increasing greenhouse gases (Slingo, 1990). Thus, climate model behavior in subtropical stratocumulus to cumulus transition regions such as the Northeast Pacific is critical in representing Earth's energy balance and cloud feedbacks on climate.

Significant biases and challenges persist in the representation of stratocumulus clouds in climate models (e.g. Hannay et al. (2009), Medeiros et al. (2012), Lin et al. (2014), Dal Gesso et al. (2015)). Traditional parameterizations have difficulty representing stratocumulus and their transition to cumulus, because the stratocumulus layer is thin, capped by a sharp inversion, and maintained by turbulent eddies unresolved at the grid scale of climate models. These eddies are strongly influenced by the clouds and their radiative effects, and may have a complex vertical structure. Turbulent entrainment through the sharp inversion is particularly important but subject to numerical errors as well as representational uncertainties. 
The complexity of this problem and the relatively slow improvement of climate model simulations of these cloudy boundary layers suggests the exploration of radically different parameterization approaches, such as 'ultraparameterization' (Parishani et al., 2017) or the use of machine learning to holistically represent the combined effect of all of the interacting processes, the approach explored here.

Here we use machine learning to emulate reanalysis. Using reanalysis allows our model to learn not only from the conventional parameterization used in the global forecast model, but also from the observational update or 'analysis increment' applied in the data assimilation cycle. This update provides a natural bias correction to the conventional model, which can improve performance compared to the parameterization suite of the forecast model used to create the reanalysis. In this study, we focus on the summertime Northeast Pacific, a particularly well-studied subtropical transition region (e.g. Stevens et al. (2003); Zhou et al. (2015); Albrecht et al. (2019)) with large horizontal gradients of sea-surface temperature (SST) and climatological low cloud cover. To speed up training, we limit the domain size and the scope of the network to be able to sufficiently sample the variability with a dataset that fits in the $4 \mathrm{~GB}$ of memory available on our GPU. However, there is no fundamental reason the same approach could not be applied globally using computational resources sufficient to process the larger volume of data, for example by using multiple GPUs training in parallel.

We use an artificial neural network (ANN) to predict the lower-tropospheric heating and moistening tendencies in each model grid column. An ANN is a nonlinear machine learning model trained by iteratively modifying a large number of model weights to optimize a loss function. ANNs have been used successfully to emulate radiation in a prognostic setting (Krasnopolsky et al., 2005) and to diagnose cloud fraction and precipitation (Krasnopolsky et al., 2013). More recently they have been used to represent convection in a prognostic setting, using deep networks on superparameterization model data (Rasp et al., 2018) and a novel approach to prognostic model training on near-global cloud-resolving model data (Brenowitz \& Bretherton, 2018).

We begin by discussing the reanalysis dataset (Section 2) and outline the MARBLE model (Section 3). This is followed by an evaluation of MARBLE's performance over the Northeast Pacific (Section 4). Finally, we summarize key strengths of MARBLE 


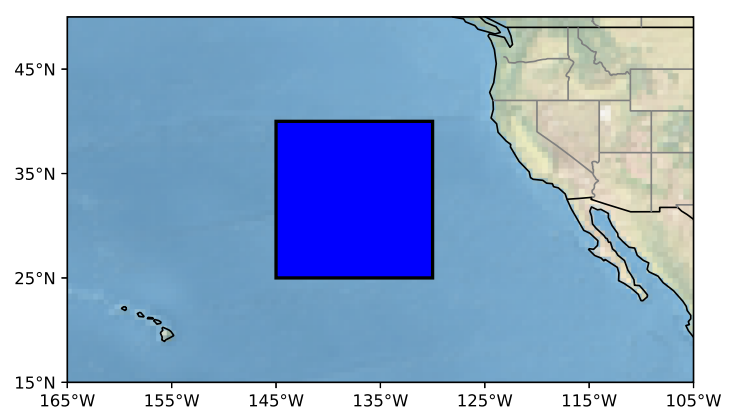

Figure 1. Study region used for model training and evaluation. The blue square indicates the region of study.

and discuss future extensions of this data-driven machine-learning parameterization approach (Section 5).

\section{Dataset}

The European Center for Medium-Range Weather Forecasts (ECMWF) Reanalysis 5th Generation (ERA5) is a global reanalysis dataset, documented at https://confluence. ecmwf.int/display/CKB/ERA5+data+documentation. In this study we use the highresolution realization (HRES) run at $31 \mathrm{~km}$ horizontal resolution, which provides hourly data on 31 model levels in the vertical domain we are modeling (from the surface to $3 \mathrm{~km}$ ). Our study uses reanalysis data for June-August 2008-2016 over the Northeast Pacific on a $0.5^{\circ}$ grid from $230^{\circ} \mathrm{W}-215^{\circ} \mathrm{W}$ and $25^{\circ} \mathrm{N}-40^{\circ} \mathrm{N}$, as shown in Figure 1.

MARBLE is trained and evaluated on hourly values in June, July, and August. For state quantities such as temperature, surface pressure, vertical wind, humidity, or mixing ratios, instantaneous values are used. For flux quantities such as surface precipitation or radiative fluxes, hourly mean values are used. Data from 2008 through 2014 are used for training, and validation is performed on 2015 data to determine a reasonable model configuration. Data from 2016 are used for evaluation once all model configuration has been determined, including number of neurons, depth of network, choice of activation function, etc. 


\section{Model Description}

Following most conventional climate model parameterizations, we use a vertically nonlocal column-oriented approach. The diabatic heating and moistening tendencies at each height within a grid column are functions of conditions throughout that grid column, but not other grid columns. Other boundary conditions at the surface or top of our simulated tropospheric layer, e. g. surface fluxes of sensible and latent heat, are allowed to affect the entire vertical column in a single time step, reflecting possible rapid vertical transport in turbulent eddies.

We apply the multi-timestep training approach used by Brenowitz and Bretherton (2018) to vertically-resolved reanalysis data over the Northeast Pacific. This training approach generates a numerically stable and accurate model, whereas a model trained on tendencies at each timestep is numerically unstable. We call this approach MachineAssisted Reanalysis Boundary Layer Emulation (MARBLE). MARBLE replaces all subgrid and radiative heating parameterizations and resolved vertical advection within its domain. For simplicity, our implementation does not yet predict momentum tendencies.

\subsection{Dimensionality Reduction}

Rather than use the full resolution vertical profile of the ERA5 product, we reduce the dimensionality of the data using principal components on a constant height grid. Like the choice of a limited horizontal region, this is done to reduce the memory footprint of the training dataset. First, the ERA5 profiles are interpolated to an evenly spaced height grid of 20 points from the lowest model level to a height of $3 \mathrm{~km}$. The mean vertical profiles based on the training data (Figure S1) are removed. The time-height perturbations of each variable are separately decomposed into principal components. We keep enough components to explain $99 \%$ of the variability of the training data - 10 components for liquid water static energy $\left(s_{l}\right)$ and total water mixing ratio $\left(r_{t}\right)$ and 5 for vertical wind (Figure S2).

Tendencies are decomposed using the principal components of their respective prognostic variable (for example, clear-sky radiative heating rates are decomposed using the basis for $s_{l}$ ). All vertically-resolved quantities are input and output as principal components. All inputs and outputs are additionally normalized so that their values in the training dataset have a mean of 0 and variance of 1 . After training, computed outputs 
are re-scaled and transformed back to a constant height grid for plotting and error evaluation.

\subsection{Neural Network Design}

The core of MARBLE is a feed-forward neural network for supervised learning. The loss functions being iteratively minimized during training are weighted sums of mean squared errors of the model outputs, as described below. They do not include any regularization terms, as L1 and L2 regularization were found not to significantly affect model generalization.

MARBLE is trained while integrating forward 7 days with a one-hour forward Euler timestep, as shown in Figure 2. Training with a significantly shorter period of 2 days was found to produce a model that is numerically unstable within a few days, whereas after training over periods of 7 days we did not observe instability in the model even after a month-long forecast. Figure 2b shows the internal structure of the neural network. This is a deep neural network with two hidden layers and uses the rectifier linear activation function, defined as

$$
R(x)= \begin{cases}x & \text { if } x \geq 0 \\ 0 & \text { if } x<0\end{cases}
$$

The input, internal, and output layers contain 33, 128, and 51 neurons, respectively. Figure $2 \mathrm{a}$ shows in detail how the neural network is used to compute the next model state in the course of training. The components are color coded to show their role in model training. Input forcings from the ERA5 dataset include surface latent and sensible heat flux, sea surface temperature, surface pressure, mid and high cloud fractions, downwelling shortwave fluxes at top-of-atmosphere and top-of-domain, top-of-domain rainwater mixing ratio, and the vertical structure of vertical wind decomposed into its principal components. The input to the network also includes the principal components of $s_{l}$ and $r_{t}$ output from the previous timestep, which on the first timestep are taken from the ERA5 dataset. These inputs include all relevant top and bottom-of-domain forcings.

The neural network produces a set of diagnostic outputs, a clear-sky radiative heating rate which is optimized to match its ERA5 counterpart, and unconstrained "residual" tendencies for $s_{l}$ and $r_{t}$. This includes effects of surface fluxes, analysis increments, 


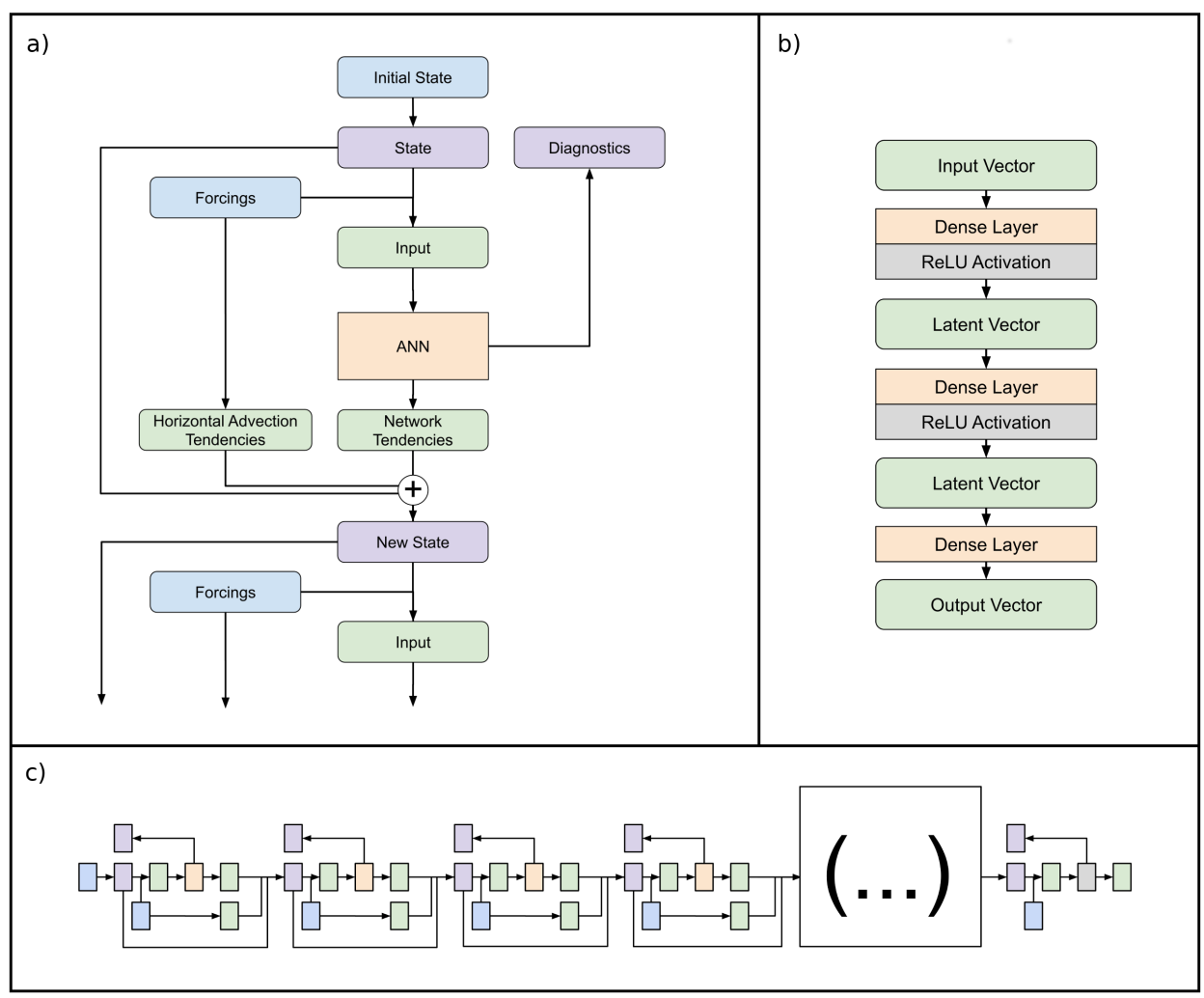

Figure 2. Artificial neural network structure and training approach, showing a) how components are connected in the first timestep, b) the internal structure of the ANN component, and c) how those components are chained together to train over multiple timesteps. Orange represents operations with trainable weights, grey represents static operations without trainable weights, purple represents output vectors whose values are included in the optimized loss function, green represents internal vectors used by the model, and blue represents data collected from ERA5 used as input to the model. At each timestep in c) the ANN uses the same weights. Network tendencies include both clear-sky radiative heating and residual tendencies. 
cloud radiative effects, and turbulence. At the inversion, there is usually substantial compensation between vertical advection (which is imperfectly represented by the reduceddimension basis used by MARBLE) and entrainment contributions to the residual tendency. Hence, we also fold vertical advection into the residual tendency.

These tendencies, together with clear-sky radiative heating and ERA5-derived horizontal advection of heat and moisture by the mean wind, are then added to the previous model state, producing a state at the next time step. The residual tendencies are not optimized to directly match any value in the ERA5 dataset. However, the model is optimized to produce thermodynamic states that match the states in ERA5, and the only way it can do this is by learning the correct form of tendency to add to the model state.

Figure 2c shows the full structure of the system being optimized. While not pictured, a total of 168 time units are used to step the model state forward a total of 7 days in 1-hour increments. In training, the system is considered as a single neural network whose optimized output includes diagnostic values and model state at every timestep (indicated by purple boxes).

The full equations for the tendencies at each timestep can be written as:

$$
\begin{aligned}
\frac{d s_{l}}{d t} & ={\frac{d s_{l}}{d t}{ }_{h a d v}}+\mathbf{W}_{s l} \cdot R\left(\mathbf{W}_{2} \cdot R\left(\mathbf{W}_{3} \cdot \vec{x}_{i n}+\vec{b}_{3}\right)+\vec{b}_{2}\right)+\vec{b}_{s l} \\
\frac{d r_{t}}{d t} & ={\frac{d r_{t}}{d t}{ }_{h a d v}}+\mathbf{W}_{r t} \cdot R\left(\mathbf{W}_{2} \cdot R\left(\mathbf{W}_{3} \cdot \vec{x}_{i n}+\vec{b}_{3}\right)+\vec{b}_{2}\right)+\vec{b}_{r t}
\end{aligned}
$$

where the subscript 'hadv' denotes a prescribed horizontal advective tendency, $\vec{x}_{\text {in }}$ is a vector containing the normalized inputs, $\mathbf{W}$ represents trained weight matrices, and $\vec{b}$ represents trained bias vectors. $R$ is the rectified linear activation function as defined in Equation 1. Note that apart from the horizontal advective forcing, the tendencies are entirely output by the ANN.

$\vec{x}_{\text {in }}$ includes all inputs to the neural network as previously described in this section, concatenated into one vector. Horizontal advective fluxes are intentionally not included in $\vec{x}_{\text {in }}$ to avoid the model learning the inversion height from the vertical structure of the ERA5 advective forcings, an approach that might go wrong in a three-dimensional simulation.

Using normalized outputs, the loss function being minimized is

$$
E=\frac{1}{N_{s} N_{t}} \sum_{\text {sample timestep }} \sum_{z_{t}} \int_{z=0}^{z=z_{t}}\left(\frac{1}{4}\left[\left(s_{l}^{m}-s_{l}^{a}\right)^{2}+r_{t}^{m}-r_{t}^{a}\right)^{2}\right]
$$




$$
\begin{aligned}
& \left.+\frac{1}{14}\left[\left(r_{c}^{m}-r_{c}^{a}\right)^{2}+\left(r_{r}^{m}-r_{r}^{a}\right)^{2}+\left(C^{m}-C^{a}\right)^{2}+\left(R_{c s}^{m}-R_{c s}^{a}\right)^{2}\right]\right) d z \\
& \left.+\frac{1}{14}\left[\left(C_{l o}^{m}-C_{l o}^{a}\right)^{2}+\left(P_{s}^{m}-P_{s}^{a}\right)^{2}+\left(\mathscr{L}^{m}-\mathscr{L}^{a}\right)^{2}\right]\right)
\end{aligned}
$$

where subscript $m$ and $a$ refer to model and actual values respectively, $N_{t}$ is number of timesteps, $N_{s}$ is the number of training samples, and $z_{t}$ is the height of the model domain. The remaining terms represent model outputs, as listed in the following section in Table 4.2. The weighting in the loss function is defined such that half of the weight is evenly distributed between the two prognostic terms, and the other half between diagnostic outputs.

Within MARBLE, $r_{t}$ and $s_{l}$ are prognostic. All other outputs are purely diagnostic and are determined directly from $r_{t}, s_{l}$, and model inputs. Because these diagnostic outputs are trained over the course of many timesteps in MARBLE, they can be accurately diagnosed from the kind of model state that MARBLE produces. In contrast, were we to train a diagnostic model using only ERA5 data as input, the model might perform poorly if MARBLE drifts away from ERA5-like states. We found that training a neural network to diagnose clear-sky radiative heating directly on ERA5 often produced unphysically high radiative heating rates when run prognostically in MARBLE, even at night.

Training was done using the AMSGrad variant (Reddi et al., 2018) of the Adam optimizer (Kingma \& Ba, 2015) implementation in Keras, with a learning rate of 0.002 and training batches of 4096 7-day single-column samples, initialized every 5 days. The ANN was trained for 500 epochs. In total, 130696 training samples are used for the final model.

\section{Evaluation}

To evaluate the model, we integrate the state forward 7 days in a single-column mode using the specified ERA5 forcings for each given testing location, initialized every 5 days. We compare with the corresponding ERA5 state and a baseline forecast, both projected onto the same reduced basis. There are 16337 7-day testing samples in the withheld 2016 data. 


\subsection{Baseline Model}

The fidelity of the ERA5 reanalysis relies heavily on the skill of the underlying ECMWF forecast model. Thus, a suitable single-column-like configuration of that forecast model provides a baseline for assessing whether any skill is added by MARBLE.

To this end, we used the first 12 hours of the short-term ECMWF forecasts available as part of ERA5. To make a 7 day 'baseline' forecast in any grid column, we accumulate the tendencies of $s_{l}$ and $r_{t}$ from 14 successive 12-hour forecasts, but not the differences between each 12 hour forecast and the initialization of the next forecast (which we interpret as approximating the analysis increments). This baseline is not useful for clouds and precipitation, which MARBLE treats as diagnostic variables.

Each time step we also re-project onto the 20-point evenly spaced vertical grid and perform physical adjustments, setting negative values of $r_{t}$ to zero and mixing any superadiabatic lapse rates to the dry adiabat, before projecting back into principal components. When performing dry adiabatic adjustment we neglect differences in layer mass for simplicity. This process of projection and adjustment increases baseline model squared correlation coefficients $\left(R^{2}\right)$ of $s_{l}$ and $r_{t}$ against vertical profiles from the testing data by about 0.05 in the latter half of 7 -day simulations.

Since the advective forcings from the short-range forecasts are almost identical to those in the ERA5 analysis, this baseline provides an analogue to the ECMWF model performance in single-column mode forced similarly to MARBLE. The most obvious way MARBLE can improve on the skill of this baseline is by learning the analysis increments.

\subsection{Results}

It is illuminating to inspect one such single column model run. We selected a simulation from the testing data at random for this purpose (Figure 3).

MARBLE does a remarkably good job of reproducing the evolution of ERA5 boundary structure and cloud properties in this 7-day period. In contrast to the baseline simulation, there is remarkably little apparent degradation of the forecast with time. We attribute this to the strong control of the boundary-layer depth and evolution by the vertical motion and horizontal advective forcings, a form of 'slow-manifold' behavior (Bretherton et al., 2010). Directly comparing the residual tendency produced by the ANN with its 

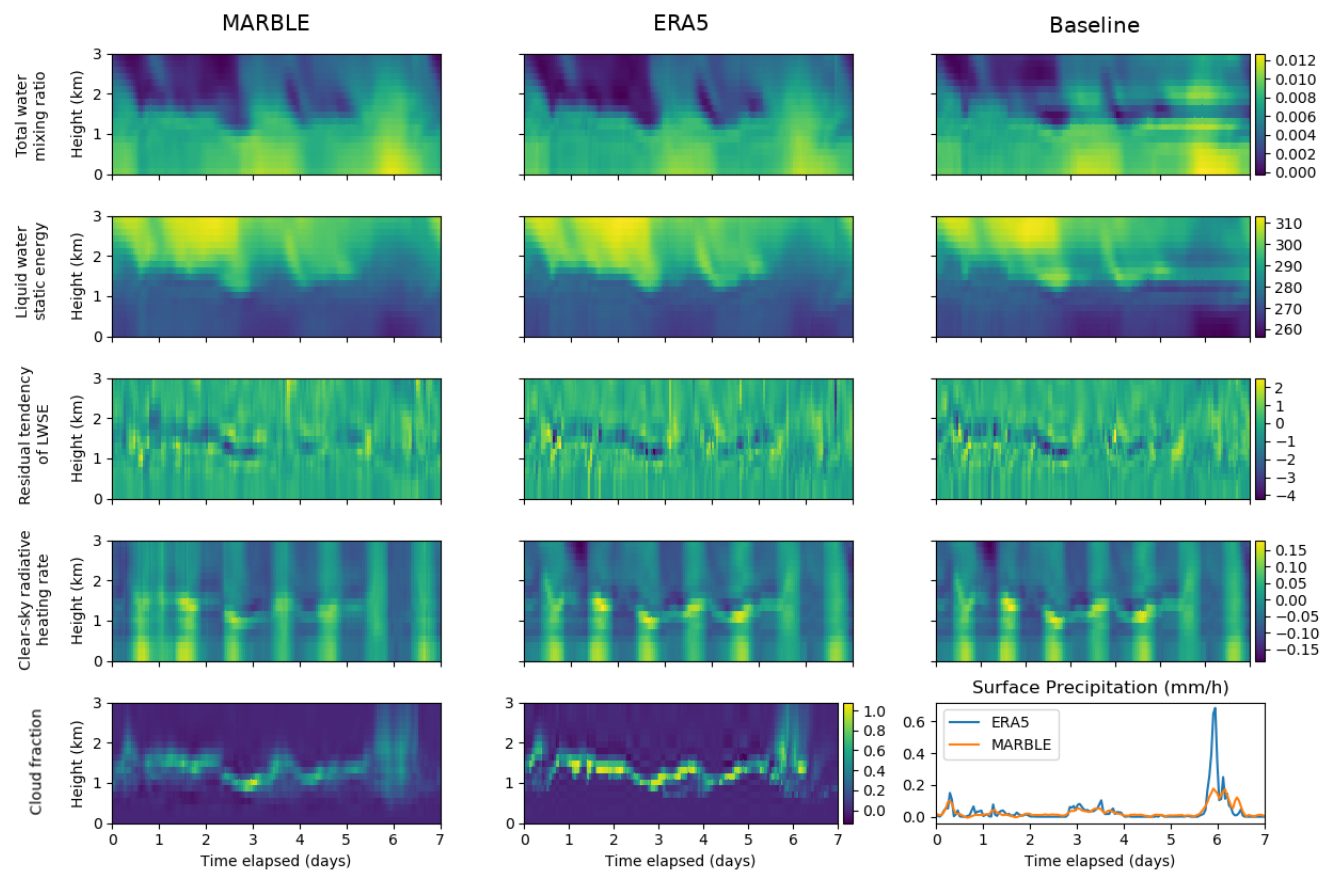

Figure 3. Randomly selected seven-day single-column prognostic simulation timeseries forced using withheld testing data, with comparison to ERA5 and baseline model. Initialized at 7:00 UTC on June 11,2016 at $30^{\circ} \mathrm{N}, 133.5^{\circ} \mathrm{W}$. Residual $s_{l}$ and $r_{t}$ tendencies have been modified by subtracting vertical advective tendencies diagnosed by centered differencing, leaving only the diabatic contributions. 
ERA5 counterpart, we see that MARBLE not only does very well at the inversion where tendencies are strongest, but also reproduces features in the tendency profile within the boundary layer and free troposphere. Inter-forecast tendencies produced every 12 hours in the analysis dataset are smoothed over longer periods in MARBLE. The clear-sky radiative heating profiles closely match the diurnal cycle of radiative heating and the impact of vertical thermodynamic structure on radiative heating rates seen in the ERA5 heating profiles.

Some values can be unphysically negative, such as cloud fraction and mixing ratios. This is due in part to the use of principal components, where the projection of the real profiles onto their components can give negative values even in the training data. For the diagnostic outputs, this can be rectified by limiting the values. For total water mixing ratio, limiting may have unforeseen negative effects since it is not active during model training.

Having seen an example timeseries, we statistically evaluate MARBLE's overall performance averaged over all NE Pacific locations and initialization times in the testing dataset. For this evaluation we use the second half of these simulations (Days 3.5-7) to avoid inheriting skill from the initial state. When we inspect model skill as a function of simulation timestep, we see the skill for $s_{l}$ and $r_{t}$ deteriorates gradually and levels off by 3.5 days (Figure $\mathrm{S} 3$ ).

MARBLE does very well at representing the thermodynamic structure and cloud properties of ERA5 over this region during the second half of the 7-day single-column forecasts, outperforming the baseline by a considerable margin. Despite its reduced vertical resolution, MARBLE has learned to improve upon the parameterized heating and moistening tendencies of the baseline ECMWF forecast model. The bias, root mean squared error, and fraction of explained variance for several quantities are listed in Table 1. As was seen in Figure 3, the largest errors are seen in vertically-resolved cloud fraction.

The horizontally-resolved mean 'climate' for JJA 2016 is shown in Figure 4. Despite not receiving any information about the spatial location of each sample other than sea surface temperature, MARBLE skillfully represents ERA5's stratocumulus to cumulus transition. There do exist small wave-like artifacts in the machine learning output, which would likely be smoothed if the columns could communicate in the horizontal. The 
Table 1. MARBLE performance for 2016 Northeast Pacific. Simulations described in Section

4. Evaluation is performed over the last 3.5 days of each 7-day simulation. The training mean at each height is subtracted before calculating the squared correlation coefficient $R^{2}$. Baseline values are computed from the baseline model described in Section 4.1. Liquid water static energy is normalized by the heat capacity of dry air at constant pressure.

\begin{tabular}{|c|c|c|c|c|}
\hline Quantity & Mean Bias & RMSE & $R^{2}$ & Baseline $R^{2}$ \\
\hline liquid water static energy $\left(s_{l} / C_{p d}\right)$ & $-0.05 \mathrm{~K}$ & $2.8 \mathrm{~K}$ & 0.81 & 0.52 \\
\hline total water mixing ratio $\left(r_{t}\right)$ & $0.065 \mathrm{~g} / \mathrm{kg}$ & $0.9 \mathrm{~g} / \mathrm{kg}$ & 0.81 & 0.54 \\
\hline clear-sky radiative heating rate $\left(R_{c s}\right)$ & $0.01 \mathrm{~K} / \mathrm{hr}$ & $0.04 \mathrm{~K} / \mathrm{hr}$ & 0.53 & $\mathrm{~N} / \mathrm{A}$ \\
\hline vertically-resolved cloud fraction $(C)$ & -0.0030 & 0.12 & 0.48 & $\mathrm{~N} / \mathrm{A}$ \\
\hline cloud water mixing ratio $\left(r_{c}\right)$ & $-1.3 \mathrm{e}-3 \mathrm{~g} / \mathrm{kg}$ & $3.4 \mathrm{e}-2 \mathrm{~g} / \mathrm{kg}$ & 0.43 & $\mathrm{~N} / \mathrm{A}$ \\
\hline rain water mixing ratio $\left(r_{\text {rain }}\right)$ & $7.0 \mathrm{e}-5 \mathrm{~g} / \mathrm{kg}$ & $2.4 \mathrm{e}-3 \mathrm{~g} / \mathrm{kg}$ & 0.92 & $\mathrm{~N} / \mathrm{A}$ \\
\hline column low cloud fraction $\left(C_{l o w}\right)$ & -0.033 & 0.18 & 0.59 & $\mathrm{~N} / \mathrm{A}$ \\
\hline surface precipitation $\left(P_{s}\right)$ & $9.8 \mathrm{e}-3 \mathrm{~mm} / \mathrm{h}$ & $8.5 \mathrm{e}-2 \mathrm{~mm} / \mathrm{h}$ & 0.74 & $\mathrm{~N} / \mathrm{A}$ \\
\hline column cloud water $(\mathscr{L})$ & $-0.0046 \mathrm{~kg} / \mathrm{m}^{2}$ & $0.026 \mathrm{~kg} / \mathrm{m}^{2}$ & 0.72 & $\mathrm{~N} / \mathrm{A}$ \\
\hline
\end{tabular}
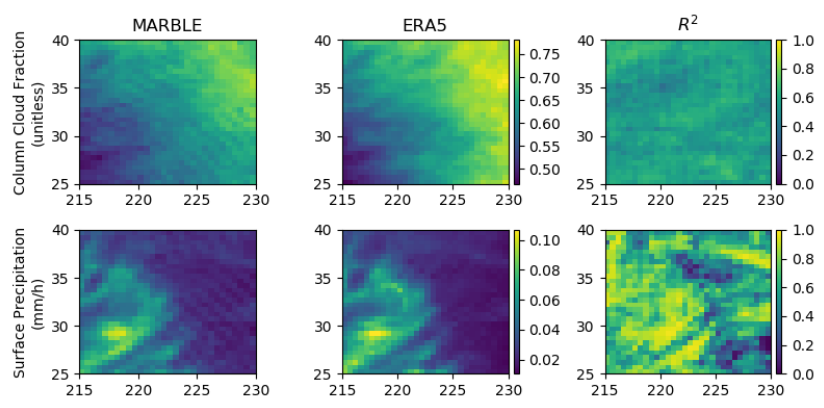

Figure 4. Horizontally-resolved mean values and correlation coefficients of key quantities, averaged across the last 3.5 days of 7-day simulations for the 2016 Northeast Pacific. 
third column in Figure 4 shows that the model does a good job of reproducing the variability of ERA5 throughout the domain.

MARBLE also reproduces ERA5's mean diurnal variability of low cloud fraction and precipitation across the NE Pacific (Figure S4). It produces both a thickening of low cloud and increase in precipitation during the night, with thinner cloud and lower precipitation during the day when cloud-top shortwave heating reduces turbulent mixing from cloud-top longwave cooling in this region.

\section{Conclusions}

We have shown that an ANN trained over many timesteps as in Brenowitz and Bretherton (2018) does a remarkably good job of matching the thermodynamic evolution and boundary layer properties of the ERA5 reanalysis dataset over the summertime Northeast Pacific in a single column model. We have created a machine learning model, MARBLE, that learns not only to reproduce the effects of the physical parameterizations in the underlying weather forecast model, but also to add the systematic impacts of analysis increments from data assimilation on the reanalysis. This serves as a natural form of model bias correction. In this study, MARBLE was trained and applied to a particular region and season. We have also successfully trained a version of MARBLE for summertime boundary layer parameterization over the southern U. S. Great Plains, and it is being tested as the lower tropospheric diabatic process parameterization for regional boundary-forced three-dimensional simulations with a mesoscale model. If this proves successful, we will try to generalize MARBLE to work globally.

\section{Acknowledgments}

We gratefully acknowledge support from DOE grant DESC0016433. Jeremy McGibbon was also supported by a Natural Sciences and Engineering Research Council of Canada (NSERC) postgraduate doctoral scholarship. We would like to thank the European Centre for Medium-Range Weather Forecasts (ECMWF) for making publically available the ERA5 reanalysis product. We would also like to thank Peter Blossey and Noah Brenowitz for helpful discussions in preparing this work, and three anonymous reviewers for helpful suggestions. Code and model weights from this study may be accessed at http:// atmos.washington.edu/ mcgibbon/mb19/. A stand-alone implementation of the model with weights can be found at https://github.com/mcgibbon/marble. 


\section{References}

Albrecht, B., Ghate, V., Mohrmann, J., Wood, R., Zuidema, P., Bretherton, C., ... Schmidt, S. (2019). Cloud system evolution in the trades (cset): Following the evolution of boundary layer cloud systems with the nsfncar gv. Bulletin of the American Meteorological Society, 100(1), 93-121. Retrieved from https://doi.org/10.1175/BAMS-D-17-0180.1 doi: 10.1175/BAMS-D-17-0180.1

Brenowitz, N. D., \& Bretherton, C. S. (2018). Prognostic validation of a neural network unified physics parameterization. Geophysical Research Letters, 45(12), 6289-6298. Retrieved from https://agupubs.onlinelibrary.wiley.com/ doi/abs/10.1029/2018GL078510 doi: 10.1029/2018GL078510

Bretherton, C. S., Uchida, J., \& Blossey, P. N. ～(2010). Slow manifolds and multiple equilibria in stratocumulus-capped boundary layers. Journal of Advances in Modeling Earth Systems, 2(4). $\quad$ Retrieved from https:// agupubs . onlinelibrary . wiley.com/doi/abs/10.3894/JAMES . 2010.2 .14 doi: 10.3894/JAMES.2010.2.14

Chen, T., Rossow, W. B., \& Zhang, Y. (2000). Radiative effects of cloud-type variations. Journal of Climate, 13(1), 264-286. Retrieved from https://doi . org/10.1175/1520-0442(2000)013<0264:REOCTV>2.0.C0;2 doi: 10.1175/ 1520-0442(2000)013〈0264:REOCTV $\rangle 2.0 . \mathrm{CO} ; 2$

Dal Gesso, S., van der Dussen, J. J., Siebesma, A. P., de Roode, S. R., Boutle, I. A., Kamae, Y., .. . Vial, J. (2015). A A single-column model intercomparison on the stratocumulus representation in present-day and future climate. Journal of Advances in Modeling Earth Systems, 7(2), 617-647. Retrieved from https:// agupubs.onlinelibrary.wiley.com/doi/abs/10.1002/2014MS000377 doi: 10.1002/2014MS000377

Hannay, C., Williamson, D. L., Hack, J. J., Kiehl, J. T., Olson, J. G., Klein, S. A., ... Khler, M. (2009). Evaluation of forecasted southeast pacific stratocumulus in the ncar, gfdl, and ecmwf models. Journal of Climate, 22(11), 28712889. Retrieved from https://doi.org/10.1175/2008JCLI2479.1 doi: 10.1175/2008JCLI2479.1

Kingma, D. P., \& Ba, J. (2015). Adam: A method for stochastic optimization. In 3rd international conference on learning representations, ICLR 2015, san 
diego, ca, usa, may 7-9, 2015, conference track proceedings. Retrieved from http://arxiv.org/abs/1412.6980

Krasnopolsky, V. M., Fox-Rabinovitz, M. S., \& Belochitski, A. A. (2013). Using Ensemble of Neural Networks to Learn Stochastic Convection Parameterizations for Climate and Numerical Weather Prediction Models from Data Simulated by a Cloud Resolving Model. Advances in Artificial Neural Systems, 2013, 13. Retrieved from 10.1155/2013/485913

Krasnopolsky, V. M., Fox-Rabinovitz, M. S., \& Chalikov, D. V. ～(2005). $\quad$ New approach to calculation of atmospheric model physics: Accurate and fast neural network emulation of longwave radiation in a climate model. Monthly Weather Review, 133(5), 1370-1383. Retrieved from https://doi.org/10.1175/ MWR2923.1 doi: 10.1175/MWR2923.1

Lin, J.-L., Qian, T., \& Shinoda, T. (2014). Stratocumulus clouds in southeastern pacific simulated by eight cmip5cfmip global climate models. Journal of Climate, 27(8), 3000-3022. Retrieved from https://doi.org/10.1175/JCLI-D -13-00376. 1 doi: 10.1175/JCLI-D-13-00376.1

Medeiros, B., Williamson, D. L., Hannay, C., \& Olson, J. G. ～(2012). Southeast pacific stratocumulus in the community atmosphere model. Journal of Climate, 25(18), 6175-6192. Retrieved from https://doi.org/10.1175/ JCLI-D-11-00503. 1 doi: 10.1175/JCLI-D-11-00503.1

Parishani, H., Pritchard, M. S., Bretherton, C. S., Wyant, M. C., \& Khairoutdinov, M. (2017). Toward low-cloud-permitting cloud superparameterization with explicit boundary layer turbulence. Journal of Advances in Modeling Earth Systems, 9(3), 1542-1571. Retrieved from https:// agupubs.onlinelibrary.wiley.com/doi/abs/10.1002/2017MS000968 doi: 10.1002/2017MS000968

Rasp, S., Pritchard, M. S., \& Gentine, P. (2018). Deep learning to represent subgrid processes in climate models. Proceedings of the National Academy of Sciences, 115(39), 9684-9689. Retrieved from https://www.pnas.org/content/ 115/39/9684 doi: 10.1073/pnas.1810286115

Reddi, S. J., Kale, S., \& Kumar, S. (2018). On the convergence of adam and beyond. In International conference on learning representations. Retrieved from https: //openreview . net/forum?id=ryQu7f-RZ 
Slingo, A. (1990). Sensitivity of the earth's radiation budget to changes in low clouds. Nature, 343(6253), 49-51. Retrieved from https://doi.org/10.1038/ 343049a0 doi: 10.1038/343049a0

Stevens, B., Lenschow, D. H., Vali, G., Gerber, H., Bandy, A., Blomquist, B., ... van Zanten, M. C. (2003). Dynamics and chemistry of marine stratocumulusdycoms-ii. Bulletin of the American Meteorological Society, 84(5), 579-594. Retrieved from https://doi.org/10.1175/BAMS-84-5-579 doi: 10.1175/BAMS-84-5-579

Warren, S., Hahn, C., London, J., Chervin, R., \& Jenne, R. (1986). Global distribution of total cloud cover and cloud type amounts over land. Retrieved from http://opensky. ucar.edu/islandora/object/technotes:444 doi: $10.5065 /$ d6gh9fxb

Wood, R. (2012). Stratocumulus clouds. Monthly Weather Review, 140(8), 23732423. Retrieved from https://doi.org/10.1175/MWR-D-11-00121.1 doi: 10 $.1175 /$ MWR-D-11-00121.1

Zhou, X., Kollias, P., \& Lewis, E. R. ～(2015). Clouds, precipitation, and marine boundary layer structure during the magic field campaign. Journal of Climate, 28(6), 2420-2442. Retrieved from https://doi.org/10.1175/ JCLI-D-14-00320. 1 doi: 10.1175/JCLI-D-14-00320.1 